\title{
The enhanced ASDEX Upgrade pellet centrifuge launcher
}

\author{
B. Plöckl ${ }^{*}$, P.T. Lang \\ Max Planck Institute for Plasma Physics, EURATOM Association., Boltzmannstr. 2, 85748 Garching, \\ Germany
}

\begin{abstract}
:
Pellets played an important role in the program of ASDEX Upgrade serving both for investigations on efficient particle fuelling and high density scenarios but also for pioneering work on ELM pacing and mitigation. Initially designed for launching fuelling pellets from the magnetic low field side (LFS), the system was converted already some time ago to inject pellets from the magnetic high field side (HFS) as much higher fuelling efficiency was found using this configuration. In operation for more than 20 years, the pellet launching system had to undergo a major revision and upgrading, in particular of its control system. Furthermore, the control system installed adjacent to the launcher had to be transferred to a more distant location enforcing a complete galvanic separation from torus potential and a fully remote control solution. Changing from a hybrid system consisting of PLC S5/S7 and some hard wired relay control to a state of the art PLC system allowed the introduction of several new operational options enabling more flexibility in the pellet experiments. This article describes the new system architecture of control hardware and software, the operating procedure, and the extended operational window. First successful applications for ELM pacing and triggering studies are presented as well as utilization for the development of high density scenarios.
\end{abstract}

\section{Introduction}

The injection of pellets into fusion plasmas is regarded as a powerful tool for fuelling the plasma as well as for ELM pacing experiments. The technical development is ongoing for more than 30 years and the development of new systems is focussed on the application at JET and ITER. ${ }^{1-3}$ Many studies were carried out for high efficiency fuelling studies and ELM pacing and confirmed the relevancy of this technology for future fusion devices. $^{4,5}$

The ASEDX Upgrade centrifuge was developed more than 20 years ago to provide a proper fuelling tool for fusion experiments. The aim was to overcome the restrictions for the gas guns about the repetition rate $(\sim 6 \mathrm{~Hz}$ at that time) at the price of a reduced pellet speed $(1200 \mathrm{~m} / \mathrm{s}) .{ }^{6}$ The System was successful implemented at the ASDEX Upgrade tokamak, injecting pellets by direct flight from the low field side (LFS). It turned out; however, the fuelling performance is much better for pellets injected from the inboard of the torus. Launching from the magnetic high field side (HFS), particle deposition and pellet penetration is supported by the magnetic field gradient. ${ }^{7-8}$ To make this finding available for the experimental operation at a technical reasonable level, at the turn of millennium the injection path was radically modified: the centrifuge was rotated about $180^{\circ}$ and the pellets were launched through a looping guiding tube. ${ }^{9}$

\footnotetext{
*Corresponding author: bernhard.ploeck1@ipp.mpg.de
} 


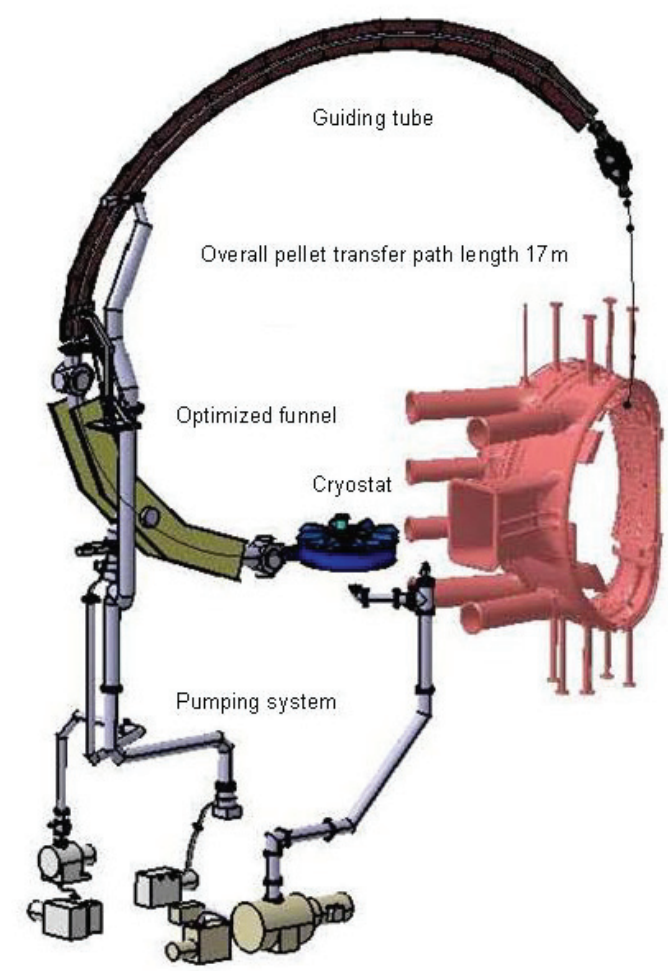

Fig. 1: Pellet injection system on ASDEX Upgrade composed of cryostats unit, centrifuge launcher, transfer looping and vacuum system placed in Seg 5. on AUG

Using this setup, several successful experiments were performed with operation focussed on pacing methods for Edge Localised Modes (ELM) and particle fuelling

Since the move of the system from the lab to the experiment beginning of the 1990ies, the control system was installed in a small room with advantageous access for cables to the torus hall. Due to some missing remote control options, this room was used as control room for the pellet system although the requested potential separation from the torus potential was not ensured. By reason of the installation of the ELM mitigation coils (Bcoils) at AUG, this place was used for its power supplies and thus the control system of the centrifuge had to move.

During 20 years of operation the control system was growing up and some hybrid systems with different technologies were generated and made the system partly confusing. Thus a simple move of control system was not possible, a substantial modernisation of the pellet injection system was essential.

The aims were:

- Get full remote control over the centrifuge system

- Remove hybrid solutions like PLC S5/S7 with some integrated relay electronics

- Reduction of hard wired electronic devices

- Introducing of field bus to reduce cabling effort

- Enhancement of vacuum performance and -safety

- Improvement of system control tools and record of system parameter options

These vast modifications were realised in the shutdown 2011/2012. The new system was successfully brought back into operation on ASEDX Upgrade during campaign 2012/2013. There were some outstanding results concerning high density scenarios as well as ELM trigger investigations in a full tungsten machine. The actual system is described, the machine parameters are presented as well as the achieved machine performance.

\section{Pellet production}

The pellet production cycle consists of two phases: 1. production of an ice rod and 2. cutting off pellets from this ice rod. Thus, the pellet production system contains of two cryostat systems: one for the ice production and one for the storage of the ice rod.

In the extrusion cryostat, a specified amount of gas will be introduced, frozen and extruded with a piston through a nozzle with a rectangular cross section into the storage cryostat. The extrusion nozzle unit contains three rectangular holes with different cross sections which are selectable by moving it to the right position. The 
temperature control of the extrusion nozzle is crucial. The extruded ice is fed into the storage cryostat; each cross section has its appropriate rectangular groove. Ice extrusion is stopped unless storage cryostat is completely filled with the ice rod (length $\sim 192 \mathrm{~mm}$ ) detected by a light barrier in the cutter unit.

In this cryostat, the ice can be stored up to $50 \mathrm{~min}$ without impact on the ice quality. The pellets will be produced at the moment, they are requested. A lever, driven by a stepping motor, pushes the ice to the cutter. The exact cutting time is derived from the position of the accelerating arm. The cutter punches the pellet out of the ice rod and it falls down to the stop cylinder. There is a small lever, which leads the pellet to the dedicated start position. This position is adjustable for good transfer efficiency. Using this stop cylinder, well defined start conditions for the acceleration through the chamfer of the arm are guaranteed. The pellet enters the chamfer of the acceleration arm and leaves it with its circumferential speed and is introduced in the transfer guiding tube (looping) by a funnel. ${ }^{10}$

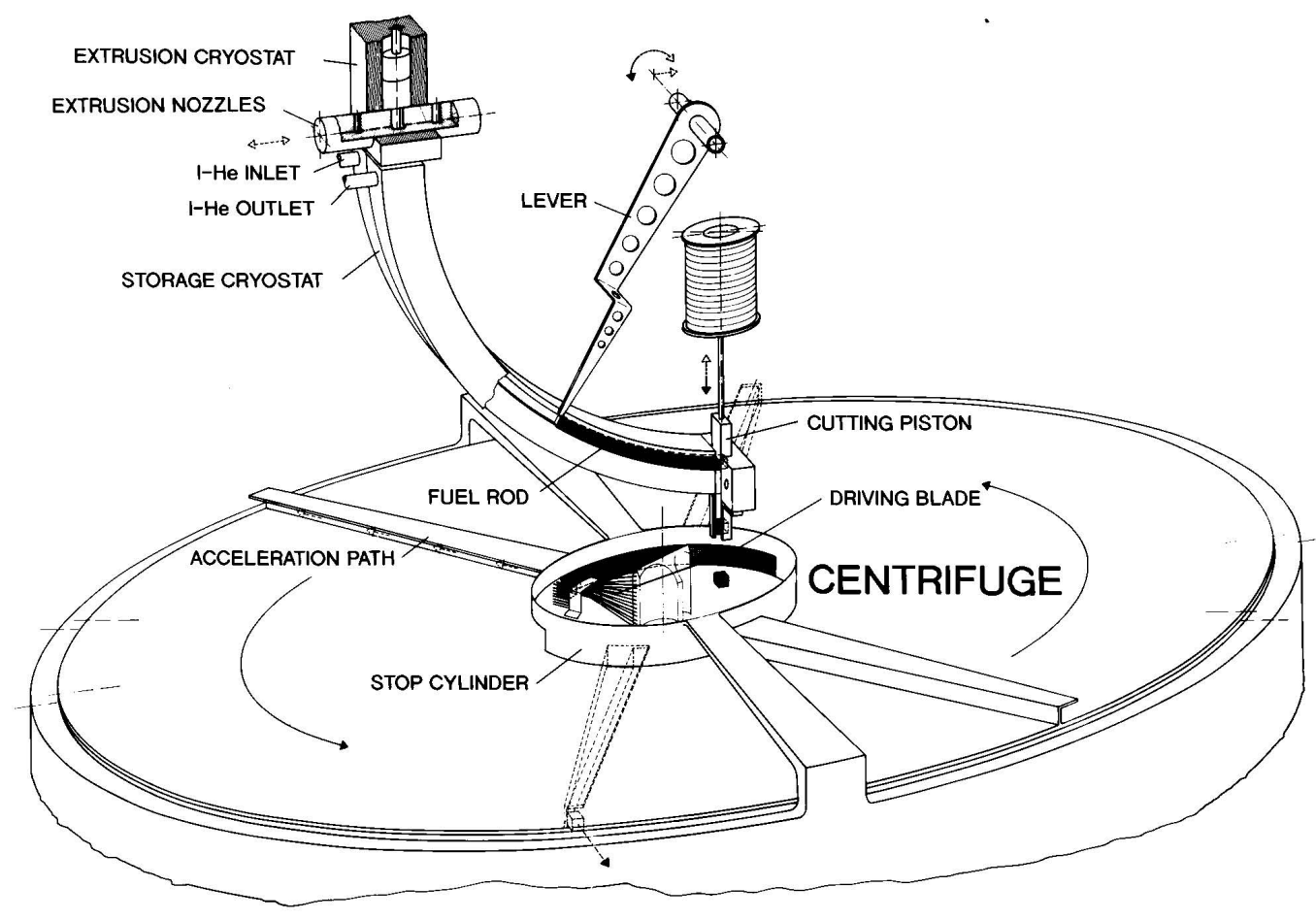

Fig. 2: View of extrusion cryostat, storage cryostat, movable 3-way nozzle, lever and cutter placed on top of centrifuge stop cylinder and acceleration arm.

The accelerating arm is mounted on the top of a big turbo pump which is backed by a roots pump as well as a rotary vane pump. The funnel and the looping sections are pumped with turbo molecular pumps. The base vacuum pressure in the system is about of $1 \times 10^{-5} \mathrm{~Pa}$.

The ice production cycle is regarded as a batch process, continuous or long pulse production of pellet launching is not possible with the actual cryostat setup. Hence, the operation costs are low compared to continuous extruders at the price of a very limited number of pellets. A study was performed to extend the cryostat to long pulse operation confirming the systems steady-state feasibility. ${ }^{11}$

\subsection{New PLC hardware and software}

The system was partly growing up through the last two decades according to the technical progress in control systems technology. This leads to a partly confusing technical situation with out-of-time constraints. Moreover, however standard in fusion experiments, there was no complete remote operation possible and the system didn't comply to potential separation rules. To overcome this situation, a substantial renewal was carried out. The PLC was migrated from a SIEMENS S5/S7 Hybrid to a full S7 system, and the functionality from a lot of discrete electronic hardware was integrated into the PLC. The cryostat temperature control electronics was replaced by PID loop (with no derivative term) implemented in the PLC program. A fast and reliable remote control of the cryostat temperature was achieved. The PLC program has to be adapted and improved. SIEMENS WinCC serves as human-machine-interface (HMI). 


\subsubsection{Integration of discrete hardware for pellet production into FM352-5}

The optimisation of the pellet production sequence is essential to achieve high repetition rates (up to $\sim 80 \mathrm{~Hz}$ ). The minimum cycle time is constituted by the time required to feed forward the ice rod, the waiting time for the synchronisation with the centrifuge arm position and the time for the cutter activity.

The feed rate of the ice rod is $0.25 \mathrm{~m} / \mathrm{s}$, (step length $0.25 \mathrm{~mm}$, motor frequency $1 \mathrm{kHz}$ ), thus the feed forward time depends of the pellet size, $6 \mathrm{~ms}$ for small size, $7 \mathrm{~ms}$ for medium size and $8 \mathrm{~ms}$ for big size pellets. The maximum time needed for synchronisation of cutter activity is the period of centrifuge revolution and depends on the centrifuge frequency and therefore on the pellet speed. At the lowest speed $(240 \mathrm{~m} / \mathrm{s})$, the time of revolution is $16 \mathrm{~ms}$, the corresponding time at a speed of $560 \mathrm{~m} / \mathrm{s}$ is $7.1 \mathrm{~ms}$. At the highest speed $(1050 \mathrm{~m} / \mathrm{s})$ the time is $3.8 \mathrm{~ms}$. The time for the cutter activity turned out to be $4 \mathrm{~ms}$.

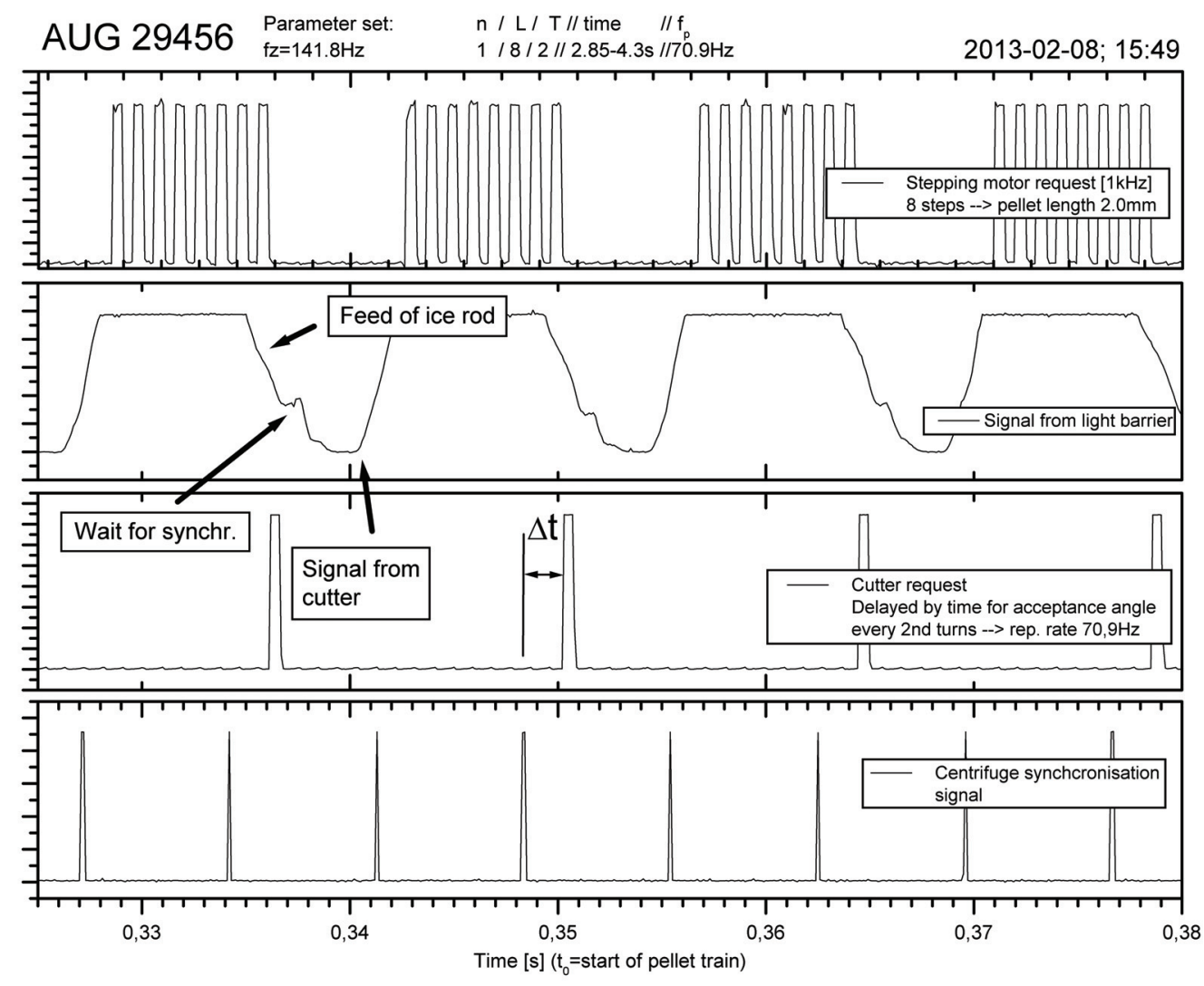

Fig. 3: Illustration of pellet production sequence. First track shows the request of stepping motor ( $1 \mathrm{kHz}$ pulses). The second track shows the signal of the light barrier, which controls the cutter box. The falling edge indicates the feed of the ice rod. Due to the transparency of the ice, the signal does not drop to zero. On the fourth track, the centrifuge synchronisation signal is displayed. The cutter activity (shown in the third track) is delayed by a certain time and repeated in respect to the requested repetition rate. The cutter blocks the laser beam, hence the diode signal drops to zero.

Taking this into account, the minimal cycle time for big pellets is $12 \mathrm{~ms}$ which corresponds to a maximum repetition rate of $83 \mathrm{~Hz}$.

As mentioned above, the timing of the pellet production sequence is a critical issue and was former realised by some hardware units, which constricted the desired flexibility concerning repetition rate and accuracy concerning number of pellets.

Following requirements were emerged from the experimental routine.

- The first request was to toggle between some certain repetition rates during the discharge to adjust $\mathrm{D}_{2}$ flux to the plasma need.

- The second request was to provide three time windows for pellet operation to start different parameter sets on different plasma discharge phases.

- The third request was the possibility to change the pellet length during pellet train.

To meet the temporal challenge given by the short time window of $12 \mathrm{~ms}$, a high speed PLC Boolean processor FM352-5 was chosen which have a cycle time in the $\mu$ s-range. Using the full capacity of this device, six data sets for pellet parameter and three time windows are well-defined. 
A data set for pellet parameters consists of number of pellets, pellet length (number of motor steps), divider (divides centrifuge revolution signal to the requested synchronisation signal for the requested repetition rate by suppressing the surplus signals) and the number of the following data set.

The time window schedules the start time (relating to local timer mark TS06), the end time and the number of the pellet parameter data set, to be executed first.

Upstream connected is a gate, which is actuated by signals to protect the AUG (interlock and pulse stop), protect the centrifuge (vacuum interlock) and enabling command of the operator, of course.

The pellet production cycle (feed forward of ice, and synchronised actuation of cutter) is reset by the cutter light barrier signal. If the time window is still open and the requested number of pellets is not yet achieved, the executions of the cycles will continue until the task is accomplished or the gate will be closed by omission of enabling conditions.

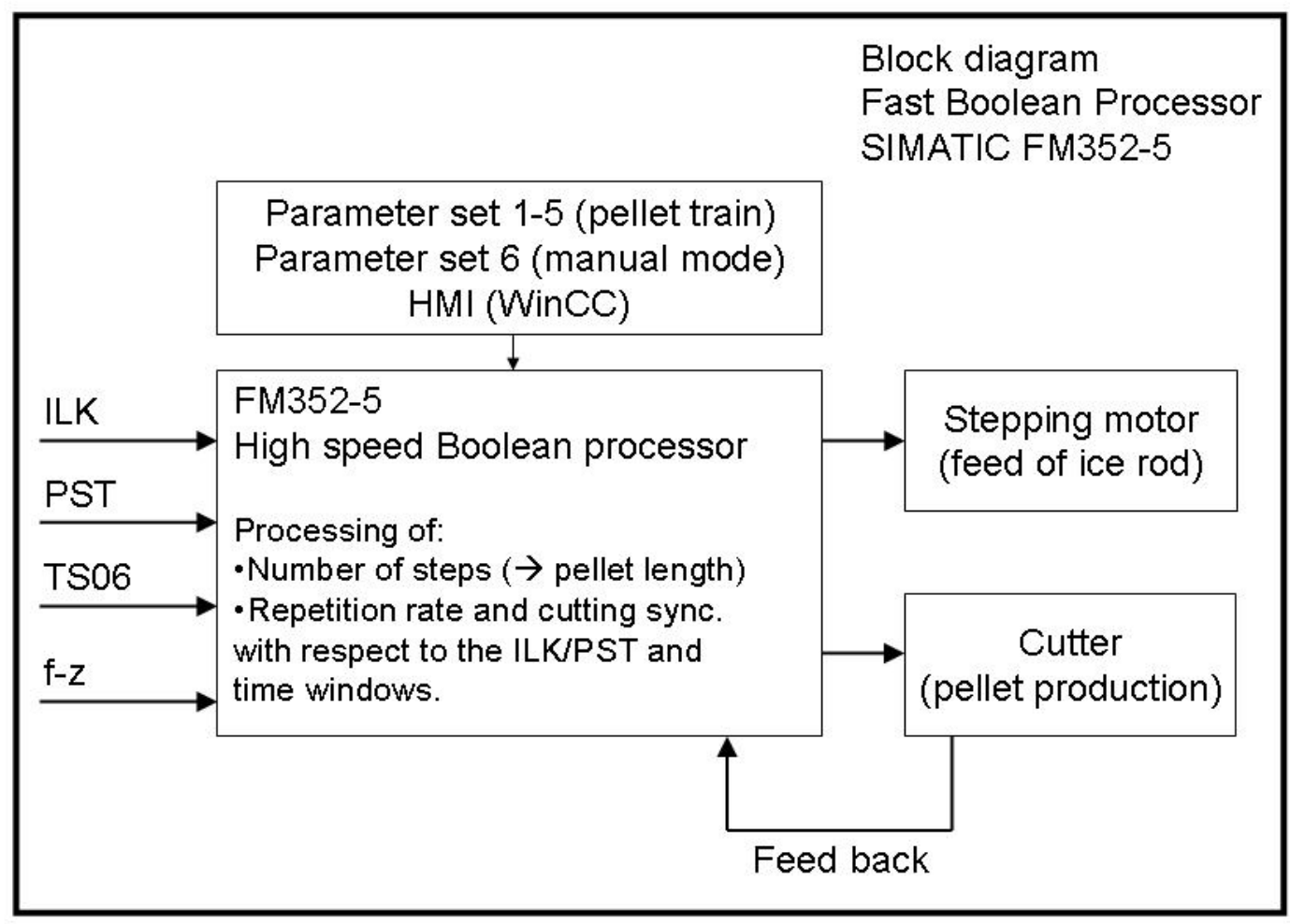

Fig. 4: Block diagram implementation of SIEMENS fast Boolean processor FM352-5. Inputs coming from left are hard wired to the processor. ILK (interlock) is derived from the plasma current, PST (pulse stop) is the AUG safety loop, TS06 marks the start of the plasma discharge and $\mathrm{f}-\mathrm{z}$ is the synchronisation signal from the centrifuge arm. The transfer of the parameter sets coming from HMI is proceed by the PLC internal (back plane) bus and has to be finished prior to start of pellet train to ensure fast execution of high speed PLC program.

\subsubsection{Integration into the AUG interlock and local timer environment}

To ensure proper and safe operation, the centrifuge control system is embedded into the AUG discharge control system via several links. The main goal is to protect the Tokamak from possible damage affected by malfunction of centrifuge system.

The gate valve to the torus is interlocked via vacuum pressure measurements.

One main task, also relevant for machine safety is a precise timing of pellet sequence. Centrifuge PLC is hard wired connected to the local timer to get the time mark. A manually activated "pellet ready" signal is sent to the machine operator to enable the launch of the countdown. During the countdown, the "last minutes workflow" has to be performed. During that time, the storage cryostat is brought from idle configuration to operation mode. The idle configuration minimises the heat load onto the ice rod. The arm is to be moved to the ice rod and some pellets are produced to get fresh ice on the front of the ice rod to compensate loss of ice length during waiting period. This procedure takes some minutes depending on the idle time (time from ice production to countdown), which may last up to 50 minutes.

The pellet injection is enabled by a signal "interlock (ILK)" which is generated as a function of plasma current. Pellet operation is enabled if plasma current is higher than $400 \mathrm{kA}$. 
The immediate shutdown of a plasma discharge is handled via a signal loop called "pulse stop (PST)" which can be actuated from every subsystem like generators, heating systems etc. A loss of this signal will stop pellet operation as well.

\subsubsection{Integration into the AUG discharge control system (DCS)}

For some control purposes, the prediction of a pellet arrival has to be provided to the discharge control system (DCS). One example successfully demonstrated, is the notching of the ECRH heating during pellet ablation. Due to the density peak during ablation, the ERCH power would be reflected on the cut-off layer and this will lead to a safety gyrotron shut down. In addition, this record is necessary to permit a subsequent analysis of the pellet train after the discharge with respect to number of fired pellets compared to arrived ones (information from ablation signal).

As a function of repetition rate and pellet speed, more than one pellet can be on the way through the guiding tube to the plasma at a given time, therefore a simple timer is not appropriate. The signal of pellet production (cutting it from the ice rod) is to be delayed by the relevant time of flight, which is composed by the time to fall down into the stop cylinder (independent of the centrifuge speed) and acceleration and flight time which are dependent on the speed. The delay is calculated as a function of number of centrifuge revolution and transferred automatically to the delay line at the moment of unblocking the gate valve to the torus vessel. As a fixing, the signal has to be $3 \mathrm{~ms}$ before the pellet arriving at the plasma to give the DCS time to react on this information.

The delay line is realized by a counter whose cycle time is equal the requested delay value. The timestamp of the incoming signal is stored by a first in first out (FIFO) method. After one cycle, the signal of the pellet production is transferred to the DCS and data acquisition system.

\subsubsection{Introduction of a field bus}

The existing system was not equipped with any field bus. Every device was hard wired to the PLC or relay control. This generates a lot of problems to ensure the potential separation from control room to torus hall.

PROFIBUS as an industrial standard was introduced in the system to act as field bus in the torus hall and connect it to the CPU which is located in the control room. All vacuum gauges, pneumatic valve units, I/O units and some turbo molecular pumps are connected to this bus, which is connected via optical link to the PLC system on the control room.

All devices, which are not able to be connected to this bus, are located in the torus hall to secure the potential separation and full remote control.

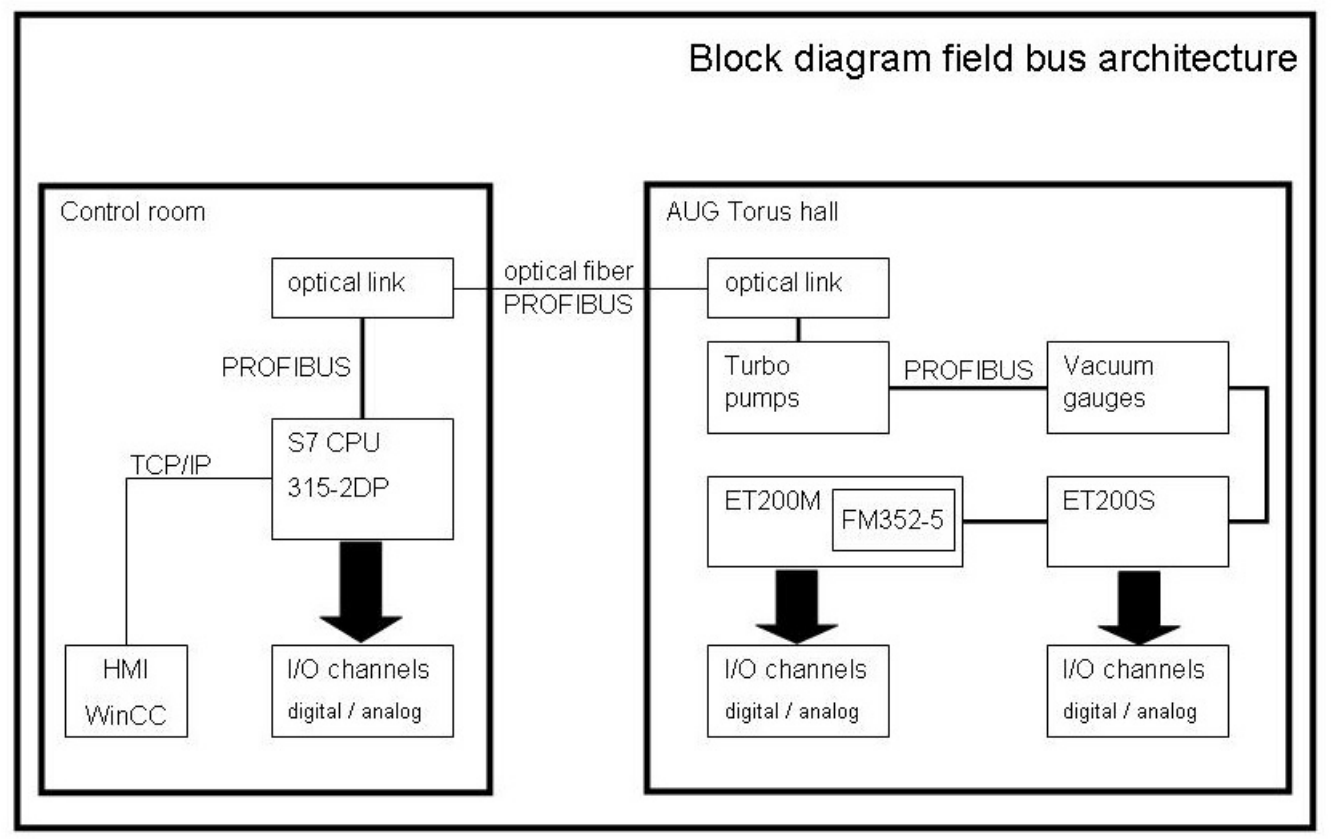

Fig. 5: Block diagram of field bus architecture. All PLC components as well as vacuum gauges and some turbo molecular pumps are connected via PROFIBUS DP. This reduces the cabling effort and enables remote control and diagnostic of components. The fast Boolean processor FM352-5 is integrated in an ET200M with direct wired I/O for fast operation. Only communication with CPU (parameter transfer) is managed via back plane bus of PLC. 


\subsubsection{Pneumatic valve unit}

A lot of electro pneumatic valves former distributed on several places of the system are merged into three compact pneumatic valve units (Buerkert) linked via PROFIBUS to the PLC. These valve units have some I/O ports to receive the response of the position indicators and distribute digital commands.

\subsection{Investigation of new functionality of pellet trains}

The parameters, which define a pellet train, are: number of pellets, pellet length, repetition rate (integer fraction of centrifuge number of revolutions) and subsequent data set. Three time windows are acting as a gate.

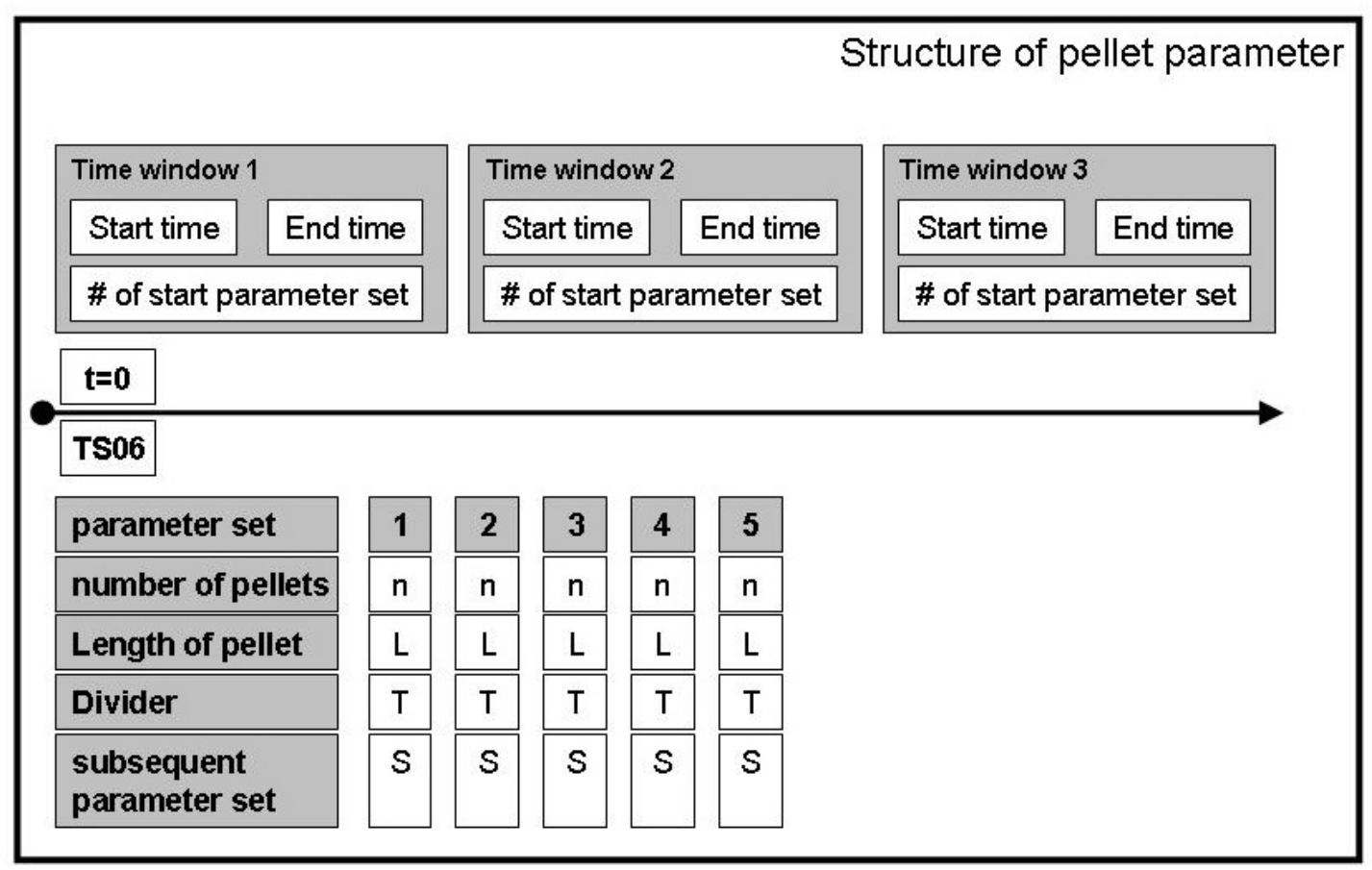

Fig. 6: Five parameter sets are available together with three time windows to create pellet trains.

The simplest operation mode is to activate one repeating data set with a time window (data set is its own successor) with number of pellets $n=1$. This operation mode is a reproduction of the previous operation mode. The time window determines the pellet operation.

The next more complex operation mode is to use a data set with a given number of pellets, ( The role of the time window is reduced to give the start time and a potentially safety stop given by the plasma scenario.

Using two or more data sets, more sophisticated patterns can be generated. The main advantage of this solution is to have well defined transitions between the data sets. This is important to ensure a minimum time interval between two cutter activities. The main application was to carry out temporal variation of material flux to the plasma in order to adapt the fuelling procedure as good as possible to the plasma need during a discharge using different repetition rates. 


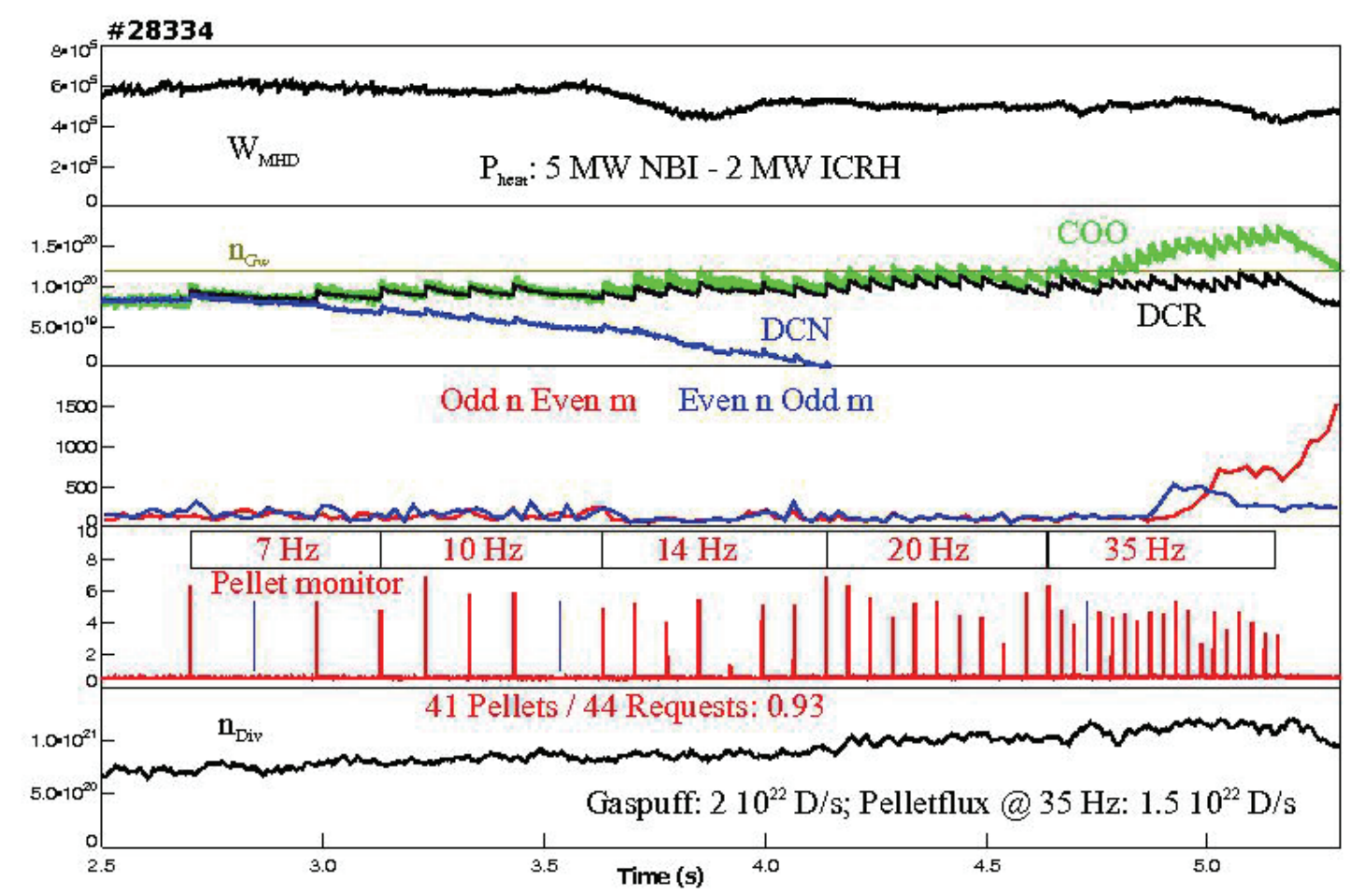

Fig. 7: An example for a smoothly stepped ramp of pellet repetition rate. Obviously an increase of the density signal is visible in the $\mathrm{CO}_{2}$ interferometer signal (COO). The DCN interferometer signal suffers from fringe loss during pellet ablation (DCN and DCR signal).

Using the option to alter the number of steps, sub size pellets can be generated. This might be useful for ELM trigger experiments, at which the fuelling effect of the pellets should be minimized as much as possible.

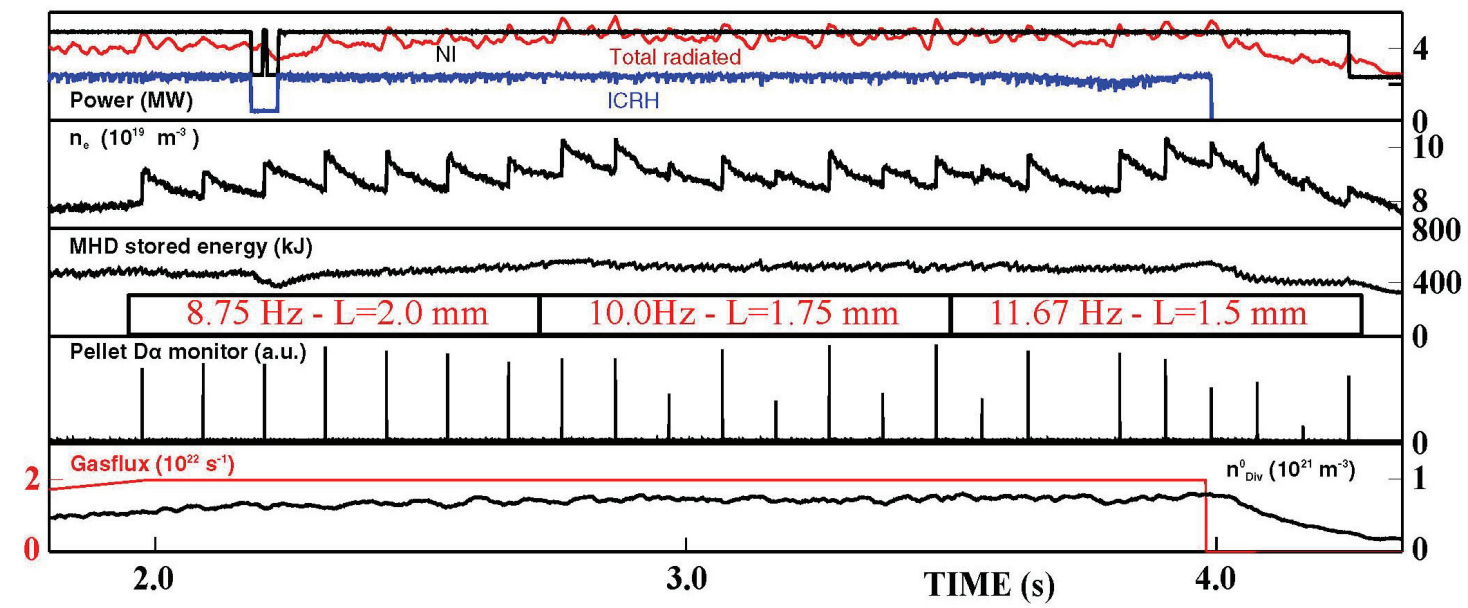

Fig. 8: Attempt to modify the pellet length. The first data set has standard length for the big cross section. The length of $2 \mathrm{~mm}$ corresponds to 8 steps of the stepping motor. In the second data set, the length is decreased by 1 step; the loss in mass flow is compensated by increasing the pellet repetition rate fro this data set $(10 \mathrm{~Hz})$. The third data set reduced the length to $1.5 \mathrm{~mm}$ and increased the repetition rate up to $11.67 \mathrm{~Hz}$.

One of the six data sets is exclusive for the so-called pre-pellet function, which is necessary in the "last minute workflow" just before a plasma discharge, but can be used for manual activation for testing, too.

The time windows are acting as gates and can be used to start pellet operation during several phases of the plasma discharge. They will stop any execution of a data set; even the number of pellets is not yet reached. Each time window can start whith an independent data set.

A wide range of pellet pattern can be realized, beginning with a simple time gate controlled execution of an infinite loop (successor is the number of the actual parameter set) up to smooth stepped ramps of repetition rate or more complex scenarios with more than one time window to perform more experiments within one discharge. 


\subsection{Enhancement of the vacuum system}

The vacuum measurement was completely adapted to PROFIBUS compatible components. A number of 14 vacuum gauges were replaced by Pfeiffer Digi Line (MPT100 / PPT100 / CPT100). The effort for wiring was significantly reduced and the control if the items and the data acquisition via PLC is efficient.

The turbo molecular pumps of the funnel and the looping were replaced by modern compound pumps: Pfeiffer HiPace 700 with TCP350 connected via PROFIBUS to the PLC. This allows full control and record of the pumps. This model has a significantly enhanced pumping speed and compression ratio for light gases compared to the previous used single stage pumps. This feature improved the pumping performance clearly.

In order to strengthen the pumping speed of the centrifuge which is basically a big turbo molecular pump (Pfeiffer TPH5000) usually running at reduced speed and therefore reduced compression rate especially for light gases, a booster pump was introduced in the fore-vacuum line. This booster decouples the pumping speed from the centrifuge speed.

Safety valves were added to all rotary vane pumps to prevent the vacuum system from oil entering the rough vacuum pumping lines. The PLC logic of the vacuum system was completely replaced. The main focus was put to ensure proper vacuum conditions, especially in case of incident.

Applying these revisions on the vacuum system a huge improvement regarding process performance was realized. In contrast to the previous configuration, no safety operation vacuum thresholds were exceeded during campaign 2012/2013, even with gas load during high pellet throughput.

\section{System parameters}

The actual specifications of the AUG centrifuge system are abstracted below.

Three different cross sections of the ice rod are available: $1.9 \mathrm{~mm}^{2} / 1.65 \mathrm{~mm}^{2} / 1.4 \mathrm{~mm}^{2}$. The nominal length of the ice rod is $192 \mathrm{~mm}$. The length of the pellet can be adjusted by steps of $0.25 \mathrm{~mm}$ length starting from the maximal particular length $(2 \mathrm{~mm} / 1.75 \mathrm{~mm} / 1.5 \mathrm{~mm})$. Standard pellets contain $4.1 \times 10^{20} / 2.7 \times 10^{20} / 1.6 \times 10^{20} \mathrm{D}-$ atoms. The mass loss during transit through the guiding tube is up to $50 \%$ depending on pellet speed and size.

\begin{tabular}{|l|l|l|l|}
\hline cross section & $1.4 \mathrm{~mm}^{2}$ & $1.65 \mathrm{~mm}^{2}$ & $1.9 \mathrm{~mm}^{2}$ \\
\hline length (nominal) & $1.5 \mathrm{~mm}$ & $1.75 \mathrm{~mm}$ & $2.0 \mathrm{~mm}$ \\
\hline $\begin{array}{l}\text { max. number of } \\
\text { pellets }\end{array}$ & 128 & 109 & 96 \\
\hline $\begin{array}{l}\text { number of atoms / } \\
\text { pellet }\end{array}$ & $1.7 \times 10^{20}$ & $2.8 \times 10^{20}$ & $4.3 \times 10^{20}$ \\
\hline $\begin{array}{l}\text { max D- flux } \\
\text { @70Hz (nominal) }\end{array}$ & $1.2 \times 10^{22} 1 / \mathrm{s}$ & $2,0 \times 10^{22} 1 / \mathrm{s}$ & $3,0 \times 10^{22} 1 / \mathrm{s}$ \\
\hline
\end{tabular}

Table 1: number of atoms and maximum flux for different pellet sizes

\section{1. $\quad$ Pellet speed}

The centrifuge consists of a big turbo molecular pump equipped with an acceleration arm. For this reason the structure is very sensitive for resonant oscillations. In order to reduce wear and tear of the bearing, the idle rotation frequency is set to $60 \mathrm{~Hz}$ as there was found a minimum of oscillating forces. Considering the resonant frequency ranges three further fixed centrifuge speed levels are pre- adjusted $(60 \mathrm{~Hz} / 110 \mathrm{~Hz} / 140 \mathrm{~Hz} / 260 \mathrm{~Hz})$. The corresponding pellet speed in $\mathrm{m} / \mathrm{s}$ is generated by multiplying the frequency by a factor of 4 . Hence the speed levels are $240 / 440 / 560 / 1040$ [m/s]. While the centrifuge is able to launch pellets up to a maximum speed of $1200 \mathrm{~m} / \mathrm{s}$, the transfer through the looping was found to restrict the maximum speed for intact pellet arrival in the plasma to about the chosen uppermost value of $1040 \mathrm{~m} / \mathrm{s}$. An option for continuous (step less) adjustment of the rotation speed is prepared and will be installed soon.

\subsection{Repetition rate}

The maximum achievable pellet repetition rate is given by the cycle duration for the pellet production process as described above. This cycle time was found to be $12 \mathrm{~ms}$ in the present used configuration, this leads to a maximum repetition rate of $83 \mathrm{~Hz} .{ }^{12}$ As a second boundary condition, the repetition rate has to be an integer fraction of the centrifuge revolution frequency. Thus the highest accessible pellet repetition rate is about $70 \mathrm{~Hz}$ under the actually given speed levels. 


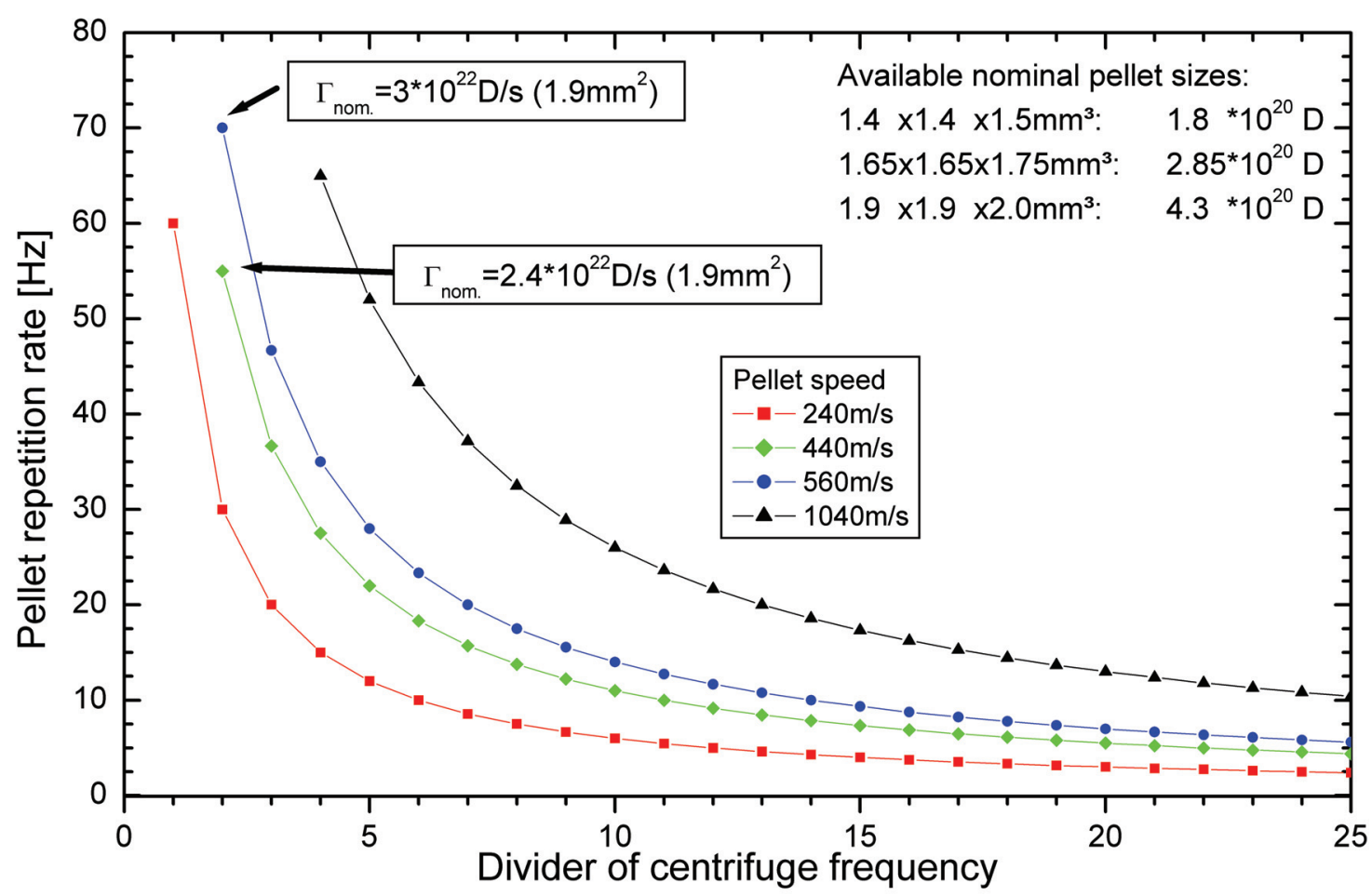

Fig. 9: Operation window of the pellet centrifuge launcher. The dotted lines show the relation between the divider of centrifuge frequency and pellet speed respectively. Every dot is an achievable setting. For two settings the corresponding mass flux is indicated.

For the repetition rates $55 \mathrm{~Hz}$ and $70 \mathrm{~Hz}$ and the biggest pellet sizes, the nominal mass flux is indicated in Fig.9. The real flux into the plasma is reduced by the mass loss in the transfer line, which is in the range of 50$80 \%$ depending of pellet size and speed.

Due to the improvements on the vacuum system, the launching of a pellet train which contains the complete ice reservoir is possible without significant increase of vacuum pressure. To avoid such pressure rise it is essential to keep stable thermal situations in the cryostat. In addition, a voltage up to $300 \mathrm{~V}$ is applied to activate the cutter. This requires caveats to avoid Paschen discharges which will damage the acting coil.

\subsection{Flight time and time jitter}

The length of the transfer guiding system is about $17 \mathrm{~m}$; the time of flight is in the range from $100 \mathrm{~ms}$ down to $20 \mathrm{~ms}$ depending on the pellet speed. The time of flight is determined from time distance between the cutter request and the pellet ablation signal in the plasma measured with a photodiode.

A well defined dependency was found with a small time jitter of typically $+/-0.3 \mathrm{~ms}$ for $\mathrm{v}_{\mathrm{p}}=560 \mathrm{~m} / \mathrm{s}$. For higher pellet speeds, the jitter is lower.

Using the delay line, the pellet arrival prediction signal is transferred to the DCS for control purposes and data acquisition system for record.

The overall delivery efficiency of the system (pellets on the plasma) turned out to be typically higher than $90 \%$ with $80 \%$ of the requested pellets showing a mass scatter of less than $10 \%$.

\subsection{Time from idle mode to operation}

The extrusion cryostat reaches its nominal working temperature after $20 \mathrm{~min}$; the storage cryostat needs 30 min. An additional time period of $25 \mathrm{~min}$. for the extrusion cryostat is necessary to get all parts cooled down to working temperature. The LHe consumption during that time is $\sim 9 \mathrm{l} / \mathrm{h}$.

\subsection{Time to change pellet speed}

The idle mode of centrifuge operation is marked by a centrifuge frequency of $60 \mathrm{~Hz}$. The ramp up to the cruising speed is performed with an increase of $4.6 \mathrm{~Hz} / \mathrm{min}$. Thus to accelerate from idle to working frequency $140 \mathrm{~Hz}$, a time span of $\sim 17 \mathrm{~min}$ is needed.

The centrifuge is not equipped with a breaking system, the retardation to a lower speed level requires more time as the rate is $1.65 \mathrm{~Hz} / \mathrm{min}$. Hence, the return from $140 \mathrm{~Hz}$ to idle frequency lasts $\sim 49 \mathrm{~min}$. This is to be taken into account planning experiments with different pellet speeds. 


\section{Application and scientific studies}

\subsection{Pacing studies}

Recently, a dedicated study on ELM pacing by pellets was performed at ASDEX Upgrade investigating the behaviour in a full tungsten (W) first wall configuration and compares it to earlier findings from experiments with at least a partial carbon wall. ${ }^{13}$ The study was encouraged by previous JET findings strongly indicating for pellet ELM pacing becoming more intricate after replacing the $\mathrm{C}$ wall by the full metal ITER like wall (ILW). The designated task of the recent ASDEX Upgrade study was to explore trigger capabilities of pellets imposing different local perturbations to the edge plasma; hence variability of pellet mass and speed was essential. Consequently, the new system was commissioned for this purpose. In order to allow for pacing studies with small fuelling burden the system settings were especially optimized for smalls sized pellets. The observed high efficiency of pellet arrival on plasma and the very low time jitter causes very reproducible and predictable pellets concerning pellet size and speed. ${ }^{14}$ This feature make the centrifuge system unique suitable for this investigations.

ELM trigger experiments were performed successfully varying the plasma perturbation by changing pellet speed and pellet size, covering in the end of the entire operational window. It was found, that with a $\mathrm{W}$ cladded wall - like in JET with the ILW and different to both tokamaks with a $\mathrm{C}$ wall - there is a lag time after an ELM where triggering of a subsequent ELM by a pellet induced perturbation cannot be achieved. The duration of this lag time can differ with respect to the plasma scenario but obviously does neither depend on pellet size nor pellet speed. Under appropriate conditions, ELM pacing was demonstrated applying high pellet repetition rate of $70 \mathrm{~Hz}$. Using the new feature of PLC to produce sub sized pellets, there was also an approach to perform net fuelling free ELM pacing. For this, the amount of pellet particles had to be restricted to the amount of particles ejected during the triggered ELM. A case where this was achieved for a short pellet sequence is shown in figure 10, displaying (from top to bottom) the evolution of the line averaged density as measured by the DCN laser interferometer, the ELM monitor signal derived from a shunt measurement of thermo currents induced in the outer divertor and the pellet monitoring signal from a wide angle diode recording the $\mathrm{D}_{\alpha}$ radiation emitted from the entire ablation region.

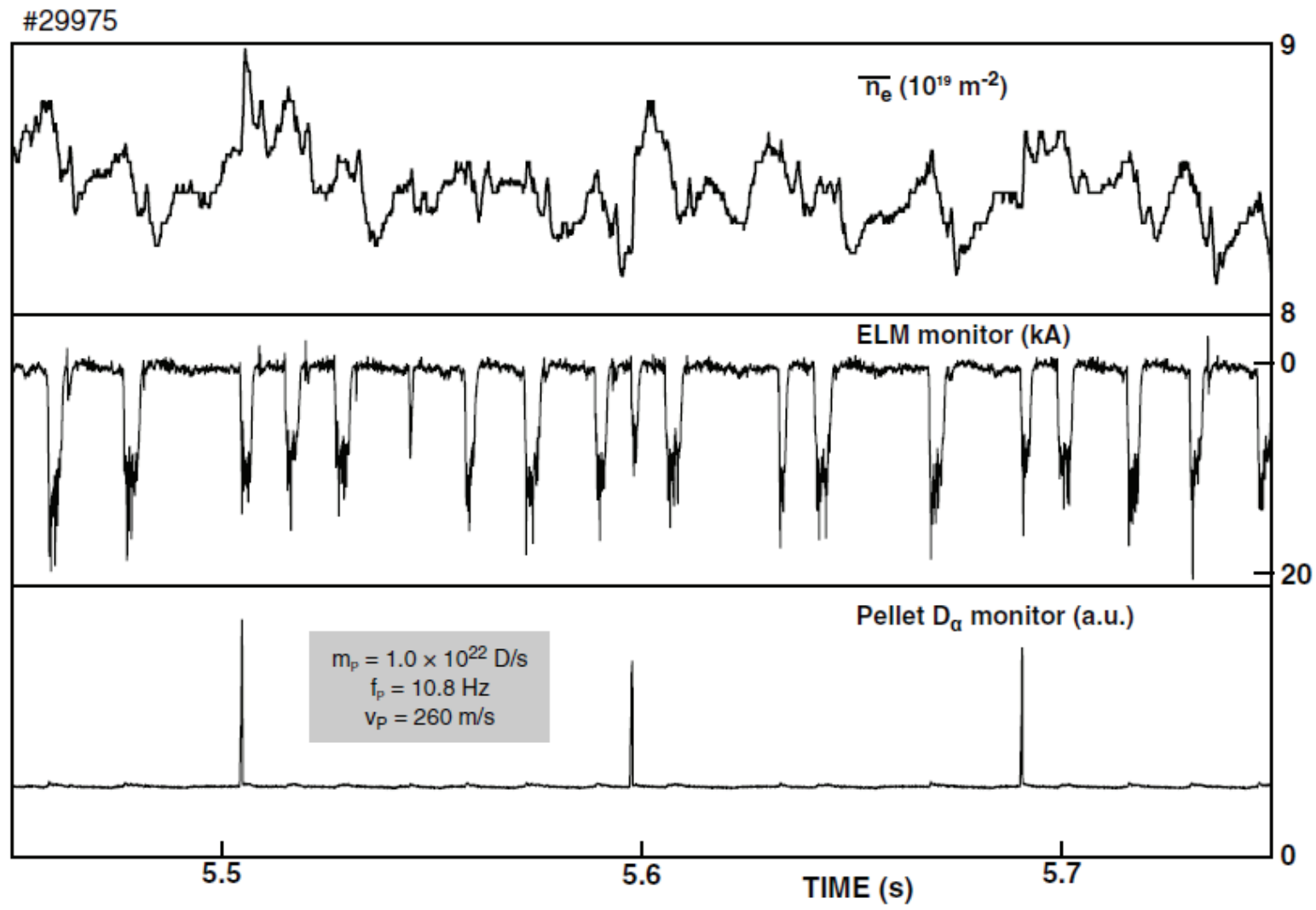

Fig. 10: Launching of sub sized pellets can still trigger ELMs but with the pellet mass about equal to the ELM induced particle losses no net fuelling is caused. 
Further effort was dedicated to experiment mimicking ELM control at ITER start up in the non-nuclear phase. The goal here was to avoid ELM free phases after the L-H transitions while the current ramp up is still on going. Since in the non-nuclear phase of ITER $\mathrm{D}_{2}$-Pellets are not suitable, $\mathrm{H}_{2}$-pellets were applied also in appropriate $\mathrm{He}$ - and $\mathrm{H}_{2}$-plasmas. The launching system is currently able to produce $\mathrm{H}_{2}$-pellets and inject them into the plasma, however reliable operation is yet restricted to large pellets.

\subsection{Fuelling operation}

The classical task of pellets injection, applied in almost tokamaks and stellarators, is particle fuelling often aiming at high density operation. In many experiments, pellet fuelling has shown a performance superior to gas puffing accessing a wider operational range. ${ }^{15}$ It is understood the system had to be ready for that operation as well. Evidently, system settings had to be adapted for delivery of large size pellets at high speed and maximized particle throughput. In particular, allowing for the maximum applied flux of about $3 \times 10^{22} \mathrm{D} / \mathrm{s}$ at a rate of $70 \mathrm{~Hz}$ enforced further optimization and fine tuning of the pellet cutting, synchronization and acceleration process. Applying such high particle fluxes, the line averaged plasma density can be increased far beyond the Greenwald limit $\mathrm{n}_{\mathrm{Gw}}$; this limit turned out to apply for the edge density only while with appropriate pellet fuelling core densities up to 4.4 times $\mathrm{n}_{\mathrm{Gw}}$ were reached. Due to the high delivery reliability and its integration in the safety system of ASDEX Upgrade the enhanced system can be operated routinely for fuelling purposes. The improved vacuum pumping capabilities turned out to be worthwhile, based on the much higher compression rate for $\mathrm{D}_{2}$. Hence the full ice reservoir can now be fired at high repetition rates without hitting any technical limits derived from heavy gas load during pellet production. An example is shown in figure 11 displaying a case where high density operation is achieved steadily for about $1 \mathrm{~s}$ until the pellet reservoir is fully consumed. Line averaged densities considerably beyond $\mathrm{n}_{\mathrm{Gw}}$ are achieved; the regime shows very benign ELM behaviour and is reversible. No loss of confinement is faced, as shown from the plasma energy and the energy confinement time calculated from absorbed heating powers and the kinetic density and temperature profiles.

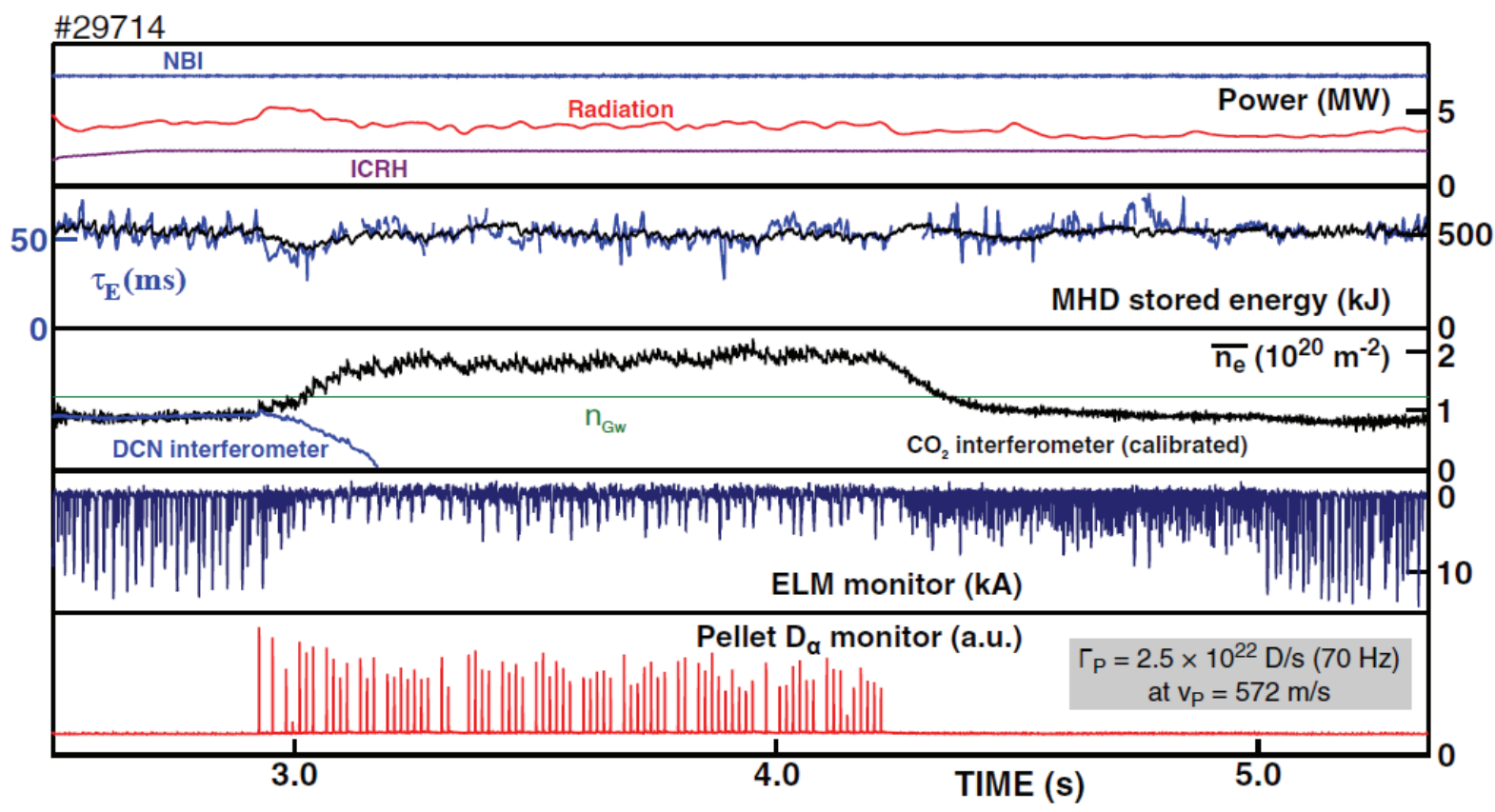

Fig. 11: Successful application of pellet fuelling to establish high density operation. Launching the entire reservoir in one go at maximum particle flux conditions achieves a line averaged density considerably beyond the empirical Greenwald limit and keeps it in steady state for more than $1 \mathrm{~s}$ until fuel runs out. A benign ELM behaviour is found in the high density regime found fully reversible and without any loss of confinement.

\section{Perspectives for steady state and tritium operation}

The presented system has still restrictions with regard to steady state operation. Using a storage cryostat the number of pellets available is of course always limited. Actually, this does not constrain significantly our experimental capabilities both for fuelling and ELM pacing studies. Nevertheless, we have already developed and presented a design of an extrusion system capable for steady state extrusion keeping all the advantages of the present centrifuge system. ${ }^{11}$ Extruders capable of long lasting (up to 1 hour) duration of operation and direct feed to the stop cylinder have been already applied at the centrifuge launcher developed at JET and JT-60U. 
Here, the almost continuous and fast extrusion of Hydrogen ice requested operation at a temperature at the extrusion nozzle significantly increased with respect to low temperatures possible when extruding relatively slow to a storage. Due to the higher pellet temperature and hence higher evaporation rates, the repulsion force acting of the pellet in the stop cylinder are much higher causing losses in accuracy and delivery reliability for the initial stop cylinder design. The problem was eliminated for the referred systems by using an improved stop cylinder setup employing a pellet guiding system which ensures the proper transfer of the pellet to the stop cylinder wall.

${ }_{16,17}$ Hence, steady state operation of a centrifuge seems well feasible in case a suitable extrusion system and a proper stop cylinder schema are applied. Originally, ITER planned to use centrifuge pellet accelerators. ${ }^{18}$

A further technical challenge met when considering operation in a future fusion test facility like ITER - the operation with Tritium (T). Although never such operated before its shut down, the JET centrifuge has been designed and predominantly constructed for the potential use of Tritium. ${ }^{19}$ Accordingly, use of Tritium can not be regarded as an obstacle for centrifuged based launching systems. Any pellet injection system is faced with mass loss from the stage of pellet production to the pellet arrival at the plasma edge. When transfer through long and considerably curved guiding tubes has to be involved they can become considerable, irrespectively of the acceleration scheme applied. Here of course the intrinsic advantage of the centrifuge principle bears fruit since no propellant gas is required potentially admixing with the ablated fuel. Consequently, direct fuel recovery from the evaporated gas becomes feasible, a significant potential relief especially when considering operation with $\mathrm{T}$. In conclusion, merging experience gained from design, manufacturing and operation of all the centrifuge based pellet systems ventured so far strongly indicates this technology is well capable to cope with requirements for operation in a fusion test facility or reactor.

\section{Acknowledgments}

The presented work was carried out in collaboration with the ASDEX Upgrade team. We would like to thank G. Sellmair and H. Eixenberger for the fruitful assistance during renewal of electronic devices, L. Kammerloher for creating the LabView application for the CompactRIO, P. Müller and N. Berger for the support to implement the PLC in the existing control system environment and W. Treutterer for the cooperation regarding DCS integration. 
[1] S.K. Combs, Pellet injection technology, Review of Scientific Instruments, vol. 64 (1993) 1697-1698

[2] A. Geraud, M. Lennholm, T. Alarcon, P. Bennett, D. Frigione, D. Garnier, P.T. Lang, A. Lukin, R. Mooney, I. Vinyar, JET-EFDA contributors; Status of the JET high frequency pellet injector, Fusion Eng. Des. (2013), in press http://dx.doi.org/10.1016/j.fusengdes.2012.12.019

[3] S.K. Combs, L.R. Baylor, S.J. Meitner, J.B.O. Caughman, D.A. Rasmussen, S. Maruyama; Overview of recent developments in pellet injection for ITER, Fusion Eng. Des. 87 (2012) 634-640

[4] P.T. Lang, G.D: Conway, T. Eich, L. Fattorini, O. Gruber, S. Günter, L.D. Horton, S. Kalvin, A. Kallenbach, M. Kaufmann, G. Kocsis, A. Lorenz, M.E. Manso, M. Maraschek, V. Mertens, J. Neuhauser, I. Nunes, W.Schneider, W. Suttrop, H. Urano, ASDEX Upgrade team; ELM pace making and mitigation by pellet injection in ASDEX Upgrade; Nucl. Fusion 44 (2004) 665-677

[5] L.R. Baylor, S.K. Combs, C.R. Foust, T.C. Jernigan, S.J. Meitner, P.B. Parks, J.B.Caughman, D.T. Fehling, S. Maruyama, A.L. Qualls, D.A. Rasmussen, C.E. Thomas; Pellet fuelling, ELM pacing and disruption mitigation technology development for ITER Nucl. Fusion 49 (2009) 085013

[6] C. Andelfinger, E. Buchelt, P. Cierpka, H. Kollotzek. P.T. Lang, R.S. Lang, G. Prausner, F.X. Söldner, M. Ulrich, G. Weber; A new centrifuge pellet injector for fusion experiments; Review of Scientific Instruments, vol. 64 (1993) 983989

[7] P.T. Lang, K. Büchl, M. Kaufmann, R.S. Lang, V. Mertens, H.W. Müller, J. Neuhauser, ASDEX Upgrade and NI teams; High efficiency plasma refuelling by pellet injection from the magnetic high-field side into ASDEX Upgrade; Physical Review Letters 79 (1997) 1487

[8] L.R. Baylor, T.C. Jernigan, S.K. Combs, W.A. Houlberg, M. Murakami, P. Gohil, K.H. Burrell, C.M. Greenfield, R.J. Groebner, C.-L. Hsieh, R.J. La Haye, G.L. Schmidt, D.R. Ernst, E.J. Synakowski, M. Porkolab; Improved core fuelling with high field side pellet injection in the DIII-D tokamak; Phys. Plasmas 7 (2000) 1878

[9] P.T. Lang, P. Cierpka, O. Gehre, M. Reich, C. Wittmann, ASDEX Upgrade Team, A. Lorenz, D. Frigione, S. Kalvin, G. Kocsis, S. Maruyama; A system for cryogenic hydrogen pellet high speed inboard launch into a fusion device via guiding tube transfer, Review of Scientific Instruments, vol. 74 (2003) 3974-3983

[10] A. Lorenz, P.T. Lang, R.S. Lang; Impact strength of cryogenic deuterium pellets for injection into tokamak plasmas; Review of Scientific Instruments, vol. 71 (2000) 3763-3743

[11] B. Plöckl P.T. Lang, G. Sellmair, J.K. Stober, W. Treutterer, I. Vinyar, ASDEX Upgrade team; The enhanced high speed inboard pellet fuelling system at ASDEX Upgrade; Fusion Engineering and Design (2013) in press, http://dx.doi.org/10.1016/j.fusengdes.2012.12.025

[12] P.T. Lang and P. Cierpka, $80 \mathrm{~Hz}$ repetitive centrifuge injector for hydrogen pellets, Review of Scientific Instruments., vol. 67, (1996) 619-620

[13] P.T. Lang M. Bernert, A. Burckhart, L. Casali, R. Fischer, O. Kardaun, G. Kocsis*, M. Maraschek, A. Mlynek, B. Plöckl, M. Reich, F. Ryter, J. Schweinzer, B. Sieglin, W. Suttrop, T. Szepesi*, G. Tardini, E. Wolfrum, H. Zohm, ASDEX Upgrade team; 40th EPS Conference on CFPP, Espoo 2013, O2.102.

[14] B. Plöckl, P.T. Lang, J. Jehl, M. Prechtl, S. Sotier, ASDEX Upgrade team, JET EFDA Contributors; Fusion Engineering and Design 86 (2011) 1022-1025

[15] B. Pégourié, Review: Pellet injection experiments and modelling, Plasma Physics and Controlled Fusion 49 (2007) R87-R160.

[16] M. J. Watson, M. Gadeberg, T. T. C. Jones, R. M. A. Lucock, V. Riccardo, J. Tait, P. Twynam, A. D. Walden, S. Wijetunge, B. Willis, Improvement, commissioning and operation of the JET pellet centrifuge, 18th SOFE 1999. p: 326 $-329$

[17] P. T. Lang, P. Cierpka, L. Horton, and A. Lorenz, M. J. Watson, P. Twynam, A. Walden, S. Wijetunge, and B. Willis N. Hosogane, H. Hiratsuka, and K. Kizu, Improved stop cylinder loading scheme for a centrifuge pellet accelerator, Review of Scientific Instruments, Vol. 71, (2000), 3744-3746

[18] S. Maruyama, Y. Yang, M. Sugihara, R. A. Pitts L. Baylor, S. Combs, S. Meitner, B. Li, W. Li, Fuelling and Disruption Mitigation in ITER, 23th SOFE 2009, p. 1-4

[19] D. J. Wilson, L. R. Baylor, P. Bennett, D. Ciric, R. Eagle, I. Hayward, A. Geraud, T. T. C. Jones, P. T. Lang, A. Lorenz, J.-L. Marecha1, R. Mooney, K. Nicholls, J. Ongena, W. Spensley, A. Thomas, M. J. Watson, Recent Developments in Pellet Fuelling at JET, 20th SOFE 2003, p. 86-91 